IIIII_2nd International

$\bar{\equiv}((((\equiv$ Electronic Conference on

$=\left(\left({ }_{11}=\right.\right.$ Sensors and Applications 15-30 November 2015

Conference Proceedings Paper-Sensors and Applications

www.mdpi.com/journal/sensors

\title{
Singular Value Decomposition Applied to Non-Cooperative Target Identification
}

\section{Olga Hernán-Vega ${ }^{1, *}$, Patricia López-Rodríguez ${ }^{1}$, David Escot-Bocanegra ${ }^{1}$ and David Poyatos-Martínez ${ }^{1}$}

${ }^{1}$ Detectability and Electronic Warfare Laboratory, National Institute for Aerospace Technology (INTA), Torrejón de Ardoz, 28850 Madrid, Spain; E-mails: hernanvo@inta.es (O.H.V.); lopezrp@inta.es (P.L.R.); escotbd@inta.es (D.E.B.); poyatosmd@inta.es (D.P.M.)

* Author to whom correspondence should be addressed; E-Mail: hernanvo@inta.es (O.H.V.); Tel.: +34-915-201-343; Fax: +34-915-202-021.

Published: 11 November 2015

\begin{abstract}
One of the main concerns in the Security and Defense area is to identify quickly and reliably different in-flight targets, especially in hostile scenarios. A non-cooperative classification system could identify targets at long range and under conditions of poor visibility without requiring aircraft collaboration. According to that, to face this problem, an approach to Non-Cooperative Target Identification based on feature extraction techniques applied to High Resolution Range Profiles is presented. The identification methodology is conducted by comparison of a collection of range profiles of a unknown target, namely test set, with a pre-loaded database of known potential signatures, namely training set. In order to evaluate the performance of the presented algorithms, range profiles are obtained through numerical simulation of seven civil aircraft at defined trajectories taken from an actual measurement campaign. The most evident issue of using synthetic signatures instead of measured profiles is that simulated profiles implies an ideal recognition scheme, since datasets have the same high quality. So as to confirm the validity of the approach, additive white Gaussian noise has been considered to the profiles in the test set.
\end{abstract}

Keywords: HRRP; NCTI; simulated/synthetic database; SVD

\section{Introduction}


A High Resolution Range Profile is the projection onto the radar line of sight of the radar energy returned by a moving aircraft. HRRP is a one dimensional image which represents the target reflectivity into the range domain. Each profile consists of a number of range bins that contain the distribution of the different reflecting parts, or scattering centers of an illuminated target, supplying information about its structure [1]. According to that, this information, different for each target, is suitable for automatic recognition since it depends on its geometry. Radar recognition has received intensive attention from the NCTI community, since it permits to recognize targets at long distance and under poor visibility conditions without the need of communication with targets and even with them being unaware of it [2]. Non Cooperative Target Identification (NCTI) by radar relies on a comparison between the measured target signatures and a reference database [3-5]. This can carried out by different techniques, including Jet Engine Modulation (JEM), HRRPs and Inverse Synthetic Aperture Radar (ISAR) images. JEM do not provide an all aspect identification capability, since the aircraft should be in an aspect angle such that the radar energy can be scattered back by the engines. Furthermore, high Signal to Noise Ratio (SNR) is desired, so the range at which aircraft can be recognized is reduced. Even if HRRP and ISAR images give an all aspect classification, the processing to obtain ISAR is very complex and time consuming, so NCTI techniques based on HRRPs are the most suitable option.

In this research, a NCTI system based on the exploitation of feature extraction techniques is presented. The process is carried out using a sequence of consecutive profiles so as to obtain information about the evolution of the position of the scattering centers along each trajectory. Two collections of synthetic HRRPs are considered, the profiles corresponding to the target to be identified, namely test set, and the ones that populate the database, namely training set.

\section{Identification Methodology}

The objective of the feature extraction techniques is to reduce dimensionality of the problem by keeping the valuable information. In this research, in order to model each aircraft as a subspace and to reduce the unsought information Singular Value Decomposition is applied to a matrix of profiles, to extract the main features of both sets. SVD is a powerful technique for the factorization of any matrix into orthogonal subspaces [6]. Let be a matrix of aligned and consecutive range profiles, $X \in$ $\Re^{N \times M}$ (assuming $N$ the number of range bins greater than the total number of profiles, $M$ ), there exists orthogonal matrices such that $X=U \Sigma V^{T}$. The columns of $U \in \Re^{N \times N}$ and $V \in \Re^{M \times M}$ are the ith left and ith right singular vectors respectively while $\sigma_{i}$ are the singular values of $X$ contained in descending order in the diagonal matrix $\Sigma \in \Re^{N \times M}$. The left singular vectors in $U, u_{i}$, span the orthogonal basis space in the range domain while right singular vectors in $V, v_{i}$, span the angle domain. Since HRRP represents the radar energy into the range domain, only $u_{i}$ will be used. The higher the value of $\sigma_{i}$, the higher the contribution of its corresponding $u_{i}$ in forming the target signal [6]. Therefore, SVD permits to define two subspaces: a signal subspace, which contains the valuable information and is spanned by the prominent singular vectors; and a noise subspace, which include the negligible data and is spanned by the rest of the singular vectors. The division into signal and noise subspace is defined by an energy threshold, $\eta=\frac{\sum_{i=1}^{K} \sigma_{i}}{\sum_{i=1}^{p} \sigma_{i}}$, in order to determine the most significant $u_{i}$ and to discard the noise subspace. This way, denoting the signal subspace as $X^{R}$ with the $K$ first $u_{i}$ of the test set and the $u_{i}^{s}$ as the $i$ th left 
singular vectors of each aircraft $s$ in the training set, the accumulated weighted angle between $X^{R}$ and $u_{i}^{s}$ is given by (1) [4]. The angle is weighted such that the singular value, $\sigma_{i}^{s}$, associated with the left singular vectors sets the significance of this angle in the final solution. Conclusively, the aircraft, $s$, of the training set that minimizes the angle between signal subspaces will be the aircraft that the radar is illuminating.

$$
F_{W}(k)=\frac{1}{\sum_{j=1}^{p} \sigma_{j}^{s}} \sum_{i=1}^{k} \sigma_{i}^{s} \cdot L\left(X^{R}, u_{i}^{s}\right) ; k=1, \ldots, K
$$

Since an energy threshold, $\eta$, is needed to define the signal subspace, the proposed algorithm $F_{W}$ involves the issue of properly define the dimensionality of the subspace. It is difficult to determine this dimensionality, so in order to avoid it, the Generalized Mutual Subspace Method (gMSM) [5] is presented. This method introduces a softweighting on the basis vectors composing the subspace. The basis are combined via this softweighting to measure the subspace similarities without definitely setting their dimensionality. Afresh, since the objective is to keep the valuable information, first, a feature extraction technique is applied to reduce the dimensionality of the problem, in this case, the eigendecomposition. The matrix $X$ can be decomposed by $X=V \Lambda V^{T}$, where $V \in \Re^{N \times N}$ is a matrix containing the eigenvectors and $\Lambda \in \Re^{N \times N}$ is a diagonal matrix containing the eigenvalues, $\lambda_{i}$. SVD and eigendecomposition are highly related; the nonzero singular values of $X$ are the square roots of the nonzero eigenvalues of $X^{T} X$ or $X X^{T}$. Both techniques allow the extraction of the main features by reducing the dimensionality. Nevertheless, the fundamental difference between them is that SVD uses two different bases, $U$ and $V$; while eigendecomposition uses just one, the eigenvectors in $V$. Once again, the higher the eigenvalue, the higher the amount of information of the target contained in its associated eigenvector. Accordingly, eigenvectors with high eigenvalues associated will belong to the signal subspace and those with low eigenvalues will form noise subspace.

While in $F_{W}$ the subspace dimensionality is defined by setting an energy threshold $\eta$, in this case the concept of softweighting tries to avoid this parameter. Softweighting gives importance to all the eigenvectors, in a way that all the eigenvalues will be weighted by a transformed value of the corresponding eigenvalues, by $\Omega=\min \left[\frac{\lambda}{\lambda_{m m}}, 1\right]$. The $m$ first values of the diagonal matrix $\Omega$ will be the unity and the rest will be proportionally decreasing with the $m$ th eigenvalue. By adding the softweighting, the importance of each eigenvector as a basis of the subspace is set. After defining the value of parameter $m$ and taking into account the diagonal matrix $\Omega$ of both sets, SVD is applied to $V_{R}^{T} \cdot V_{S}$ as in (2). Where $Q_{R}^{\prime}, Q_{S}^{T^{\prime}}$ are the matrices of singular vectors and $\Theta_{R S}^{\prime}$ is the singular value diagonal matrix whose elements represents the canonical angles' cosines.

$$
\Omega_{R} \cdot V_{R}^{T} \cdot V_{S} \cdot \Omega_{S} \quad \stackrel{S V D}{\longrightarrow} \quad Q_{R}^{\prime} \cdot \Theta_{R S}^{\prime} \cdot Q_{S}^{T^{\prime}}
$$

Here, the similarity measure will determined by the squared trace of $\Theta_{R S}^{\prime}$, that is $s_{R S}=\operatorname{tr}\left(\Theta_{R S}\right)^{2}$. The higher the $s_{R S}$, the higher the similarity between sets. Thus, the algorithm identifies the aircraft of the database, $s$, as the one with the highest similarity.

\subsection{Data sets}


Two sets from 7 civil aircraft in different flightpaths (Table 1) are used to the identification system. Test and Training samples are synthetically obtained with a computer software for all the orientations (aspect angles) contained in the flightpaths. In this research, HRRPs are generated by FASCRO [7], which applies high frequency techniques (Physical Theory of Diffraction, PTD, and Physical Optics, PO), to find the Radar Cross Section (RCS) of a target. The estimated trajectories come from a civil measurement campaign called ORFEO [8]. A total number of 22 trajectories are divided into frames for classification. Each frame (sequence of simulated profiles ordered in time) cover approximately $2.5^{\circ}$ in azimuth of the aircraft aspect angle. After the split of the trajectories, the test and the training sets are defined as matrices of HRRPs of size $N \times M$ with $N=324$ number of range bins and $M$ number of profiles, depends on the trajectory and frame chosen. The principal hesitation of using synthetic profiles instead of measured HRRPs is that simulated profiles imply an ideal recognition scheme, since datasets have the same high quality. In order to assess the robustness, additive white Gaussian noise (AWGN) has been added to the profiles in the test set.

Table 1. Dimensions of aircraft for the RCS prediction.

\begin{tabular}{lccccccc}
\hline & B747-400 & B767-300 & A310-300 & MD88 & Fokker 100 & B737-500 & Fokker 28 \\
\hline Length (m) & 70.66 & 54.22 & 46.66 & 45.10 & 35.53 & 31.10 & 29.61 \\
Wingspan (m) & 64.44 & 47.52 & 43.90 & 32.80 & 28.08 & 28.90 & 25.07 \\
Height (m) & 16.79 & 14.77 & 12.74 & 7.43 & 6.58 & 9.73 & 6.62 \\
\hline
\end{tabular}

\section{Experimental Results}

Experiments are carried out by comparison of each aircraft and frame (test set) with the database for the same frame (training set). The results are assessed and discussed here in order to define the system as accurately as possible. The main information between the 7 aircraft are contained in their respective HRRPs; normalization L2-Norm is applied to both sets before the classification takes place to ensure all profiles have comparable magnitudes.

In order to define the signal subspace dimensionality, the parameters $\eta$ for $F_{W}$ and $m$ for gMSM, have been studied to obtain the optimum values. As stated, if a high $\eta$ is chosen almost all the $u_{i}$ will be considered as signal subspace, and thus, it may contain data which in fact, is part of the noise subspace. In the same way, if $m$ is very high, more $u_{i}$ are treated with a softweight of 1 . Singular vectors representing the noise subspace will be probably considered as part of the signal subspace, thus, recognition performance can be negatively affected. Accordingly, in this research, the chosen threshold for $F_{W}$ is $\eta=0.90$ and the parameter $m$ for gMSM is $m=1$. These values offer a trade-off between the signal subspace and the recognition rates.

Considering the synthetic nature of the data sets, two case studies are presented in this paper so as to validate the performance in different identification schemes. Firstly, if comparison of simulated HRRPs is carried out, $F_{W}$ and gMSM give a great success rate (100\%) since both sets have the same nature with a very clean signature. The most evident uncertainty is the use of synthetic profiles in both datasets, since this implies an ideal recognition scheme. In order to study the feasibility of the system, white Gaussian 
noise has been added to the profiles in the test set. This way, a more real approach is simulated since profiles in the test set are observably different from those in the database. An illustration of the difference can been seen in Fig. 1 where a HRRP of an A310-300 with $\infty d B$ (no noise) and $-10 d B$ of SNR are depicted. Recognition rates for different cases of SNR are studied with the aim of demonstrating the robustness of each algorithm. A lower SNR means that more differences exist between both sets; the dominant scattering centers are reduced and additionally, when the noise is so prominent, it can be even confused with scattering centers.

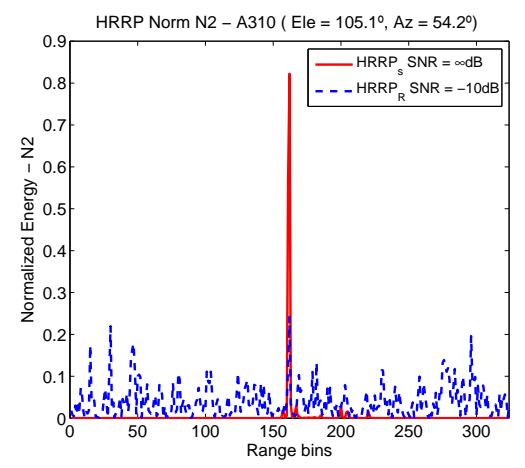

Figure 1. Variation between test and training sets

The recognition rates of 22 trajectories split into frames with a threshold of $\eta=0.90$ and a value of $m=1$ applying $F_{W}$ and gMSM, can be seen in Table 2. As expected, if no noise is added ( $S N R=$ $\infty d B$ ) the methodology gives a success rate of $100 \%$, since datasets have the same SNR. The greater the SNR, the greater similarities between both sets are obtained. This way, better recognition results are obtained $\left(98.88 \%\right.$ for gMSM and $98.48 \%$ for $F_{W}$ ), since more scatterers are visible. However, a lower SNR means that more unwanted information is present. In this study, the results are not severely influenced by noise; nevertheless when noise is highly corrupting $(S N R=-10 d B)$, it causes a decrease in the recognition rates $\left(91.63 \%\right.$ for $\mathrm{gMSM}$ and $87.48 \%$ for $\left.F_{W}\right)$. This means that, for this classification experiment, better recognition rates are obtained by applying softweighting. In fact, in this case, the classification performance has been improved $4 \%$ approximately by applying the gMSM algorithm. This means not only a progression in the recognition results, but also encourages us to keep validating the methodology presented in a real possible scenario.

Table 2. Recognition rates (\%)

\begin{tabular}{ccccccccc}
\hline & $\infty \mathbf{d B}$ & $\mathbf{1 0 d B}$ & $\mathbf{5 d B}$ & $\mathbf{3 d B}$ & $\mathbf{0 d B}$ & $\mathbf{- 3 d B}$ & $\mathbf{- 5 d B}$ & $\mathbf{- 1 0 d B}$ \\
\hline$F_{W}$ & 100 & 98.48 & 96.79 & 96.23 & 94.62 & 92.46 & 90.69 & 87.48 \\
$g M S M$ & 100 & 98.88 & 98.15 & 98.63 & 97.11 & 96.23 & 93.98 & 91.63 \\
\hline
\end{tabular}

\section{Conclusions}

In this research, two metrics for NCTI have been presented and compared, $F_{W}$ and gMSM. As demonstrated, the main objection of evaluating an algorithm with simulated profiles is the ideal 
environment, since synthetic profiles have a very clean signature and real profiles will suffer from unwanted effects such as noise or clutter. In order to validate the feasibility of the recognition approach, additive white Gaussian noise to the test set has been applied. By introducing softweighting in the gMSM algorithm, identification is achieved with no need of previously setting the subspace dimension unlike the algorithm $F_{W}$, where an energy threshold is needed to define the signal subspaces. With the softweighting, an increase in the identification rates has been accomplished comparing with $F_{W}$. Considering the difference between the samples when additive white Gaussian noise is added to the test set, the identification results presented are promising and encouraging. Nevertheless, in an actual scenario, not only noise is present but also other effects. Thus, future experiments with larger sets and with additional effects will be conducted to assure the precision of the method proposed here using measurements of real targets signatures to perform classification.

\section{Conflicts of Interest}

The authors declare no conflict of interest.

\section{References}

1. Wehner, D.R. High-Resolution Radar; 2nd ed.; Artech House, 1995; 197-237.

2. Cohen, M.N. An Overview of radar-based, automatic, noncooperative target recognition techniques. IEEE International Conference 1991, 29-34.

3. Chen, L.; Chen, X. A new radar target recognition method based on complex high resolution range profiles. Microwave and Millimeter Wave Technology (ICMMT), International Conference $\mathbf{2 0 1 2}$, $2,1-4$.

4. López-Rodríguez, P.; Escot-Bocanegra, D.; Fernández-Recio, R.; Bravo, I. Non-Cooperative Target Recognition by Means of Singular Value Decomposition Applied to Radar High Resolution Range Profiles. Sensors 2015, 15.1, 422-439.

5. Kobayashi, T. Generalized Mutual Subspace Methods for Image Set Classification. Computer Vision - ACCV 2012, 7724, 578-592.

6. Golub, G.H.; Loan, C.F.V. Matrix Computations; Third ed.; The Johns Hopkins University Press: Baltimore, USA, 1996; 69-75.

7. Perez, J.; Catedra, M.F. Application of physical optics to RCS computation of bodies modeled with NURBS surfaces. Antennas and Propagation, IEEE Transactions 1994, 42, 1404-1411.

8. Heiden, R.V.D.; Vries, J. The ORFEO Measurement Campaign; TNO Defence, Security and Safety Report, 1996

(C) 2015 by the authors; licensee MDPI, Basel, Switzerland. This article is an open access article distributed under the terms and conditions of the Creative Commons Attribution license (http://creativecommons.org/licenses/by/4.0/). 